\title{
Anger Mediates the Relationship between Pain and Depression in Irritable Bowel Syndrome
}

\author{
Mihaela Fadgyas Stanculete ${ }^{1}$, Octavia Căpățînă ${ }^{1}$, Cristina Pojoga ${ }^{2,3}$, Teodora Surdea-Blaga ${ }^{4}$
}

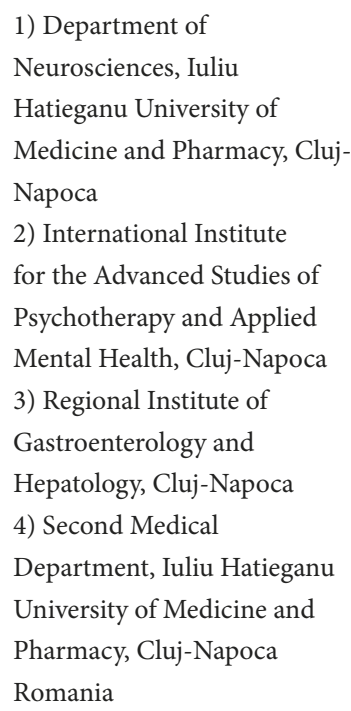

1) Department of

Neurosciences, Iuliu

Hatieganu University of

Medicine and Pharmacy, Cluj-

Napoca

2) International Institute

for the Advanced Studies of

Psychotherapy and Applied

Mental Health, Cluj-Napoca

3) Regional Institute of

Gastroenterology and

Hepatology, Cluj-Napoca

4) Second Medical

Department, Iuliu Hatieganu

University of Medicine and

Pharmacy, Cluj-Napoca

Romania

\section{Address for correspondence: \\ Octavia Capatina \\ Department of Neurosciences, Iuliu Hatieganu University of Medicine and Pharmacy Cluj- \\ Napoca, Romania \\ o.capatina@yahoo.com}

Received: 22.10 .2019

Accepted: 30.11 .2019

\section{ABSTRACT}

Background \& Aims: Anger has been linked to irritable bowel syndrome (IBS), but the mechanism underlying this relationship is still unknown. This cross-sectional study explores the possibility that anger mediates the relationship between pain severity and depression in IBS patients.

Methods: The sample included 70 consecutively evaluated patients who met Rome III criteria for IBS diagnosis and 55 healthy controls. All participants completed measures of depression, pain, state and trait anger, and anger expression style.

Results: Using a mediational model involving a series of linear regressions we found that trait anger and anger expression index partially mediated the association between depression and abdominal pain severity. The IBS patients with significant pain reported higher depressive symptoms; these results could be partially explained by their tendency to experience angry feelings generated by pain.

Conclusions: The relation between pain and depression is not a direct and linear one, but works partly through patients' tendency to become angry and through anger expression style regarding their pain.

Key words: anger - chronic pain - depression - irritable bowel syndrome.

Abbreviations: AINDEX: anger expression index; BDI: Beck depression inventory; IBS: irritable bowel syndrome; MOS: Measures of Quality of Life Survey; 36-SF-BP: 36 items Short-Form Health Survey, body pain subscale; STAXI: state-trait anger expression inventory; SANG: state-anger; TANG: trait-anger.

\section{INTRODUCTION}

Irritable bowel syndrome (IBS) is a chronic condition characterized mainly by abdominal pain and alteration of bowel movements that can substantially reduce the quality of life and work productivity, and increase the health-care costs [1-3]. Despite extensive research, the etiology of the syndrome is still unclear. Various mechanisms have been proposed. The biopsychosocial model of IBS suggests the interplay between motility, increased permeability, sensory abnormalities, alterations in the central nervous system processing of visceral pain and psychosocial factors [4-6].
Psychosocial factors have a significant influence on the onset, course, and outcome of IBS particularly in more severely affected individuals [7]. Anger is a negative affect associated with a multitude of adverse health consequences. Gate control theory and neuromatrix theory suggest that anger increases pain by altering descending and central pain modulation systems [8]. Another theory proposes that angerrelated variables may overtax the body's endogenous opioid mechanism, being less effective in managing persistent pain. Research suggests that different dimensions of anger (angerout and anger-in) are related with sensitivity to chronic pain $[9,10]$. A proposed rationale for this relationship is that IBS patients may have poor coping skills with stressful events and negative emotions [11].

Moreover, the chronic pain from IBS and the depression seem to have a bidirectional association: depression is a predictor of chronic pain, and the pain is a predictor of the persistence of depression [12]. Although the impact of negative emotions in gastrointestinal functioning has been documented in previous studies, there are still scarce data addressing this issue [13]. The evidence for the severe impact of IBS on quality of life points toward the need for the identification of 
psychological stressors in an attempt to reduce the negative consequences [14].

We elected to study the effect of anger on IBS patients because, among emotions, it is widespread in the general population. There is an important variation in psychosocial variables such as attachment style, catastrophizing, and negative beliefs depending on the geographic location. Patients from Romania are characterized by a fearful-avoidant attachment style, more catastrophizing, and negative pain beliefs [15]. Anger could be an important risk factor for adverse pain outcomes in this population.

The primary aim of this study was to determine whether there is a difference between anger state, anger trait, and anger expression between IBS patients and controls. The secondary aim assessed a possible association between chronic pain, depression, and anger. We hypothesized that anger might act as a mediating variable between chronic pain and depression in IBS patients.

\section{METHODS}

\section{Study population}

The study population included two groups and the age ranged between 18 and 70 years old. The first group consisted of 70 patients diagnosed with IBS according to ROME III criteria. The convenience sampling method was used to recruit the participants. The patients were diagnosed with IBS by a gastroenterologist and were referred to a psychiatrist for further evaluation. The three types of IBS were present in the study population: IBS with diarrhea (51.4\%), IBS with constipation (30\%) and mixed IBS (18.6\%). The control group included 55 healthy volunteers matched for age, gender, and level of education.

This study was approved by the Ethics Committee of Iuliu Hatieganu University of Medicine and Pharmacy. All procedures of this study were thoroughly explained to each participant and complied with the Helsinki Declaration of 2008. Before the study began an informed consent was obtained from each participant.

\section{Assessment instruments}

Participants from both groups were informed about the study protocol and were asked to complete a sociodemographic and clinical information form and the State-Trait Anger Expression Inventory, second edition (STAXI-2). Depression and chronic pain were evaluated in the IBS group using the Beck Depression Inventory (BDI) and subscale for Body Pain from the 36-Item Short-Form Health Survey (SF-36-BP), which is the short version of the Measures of Quality of Life Survey (MOS).

For sociodemographic and clinical information a semistructured interview was used to collect the following data: age, gender, level of education, marital status, employment status, age of IBS onset, duration and type of disease.

STAXI-2 is a 57 -item scale composed of three parts: StateAnger (SANG), Trait-Anger (TANG), and Anger Expression. It consists of six scales, five subscales, and an Anger Expression Index (AINDEX). STAXI-2 measures five domains of anger: SANG (intensity of anger feelings at some point in time),
TANG (disposition to feel anger), Anger Expression-In (frequency with which angry feelings are suppressed), Anger Expression-Out (frequency with which angry feelings are expressed), and Anger-Control (frequency with which a person attempts to control anger expression). Anger Expression-In, Anger Expression-Out, and Anger-Control are the constitutive scores of AINDEX. Participants were asked to respond to the items using a 4-point scale (1-Not at all to 4-Almost always).

The BDI-II was developed in 1996, and it is a self-report questionnaire used to evaluate the existence and severity of depression. BDI has been widely used to assess depression among patients with gastrointestinal disorders. It consists of 21 items. Participants are asked to respond using a 3-point Likert scale for each item, and the higher total scores indicate more severe depressive symptoms.

The Body Pain subscale, one of the eight subscales from 36-SF,contains two items that measure the intensity of the pain, and the way pain interferes with daily activities in the last month. The scores can vary from 2 to 12 . Each subscale score is directly transformed into a $0-100$ scale on the assumption that each question carries equal weight, and lower scores mean more disability.

\section{Statistical analysis}

The data were analyzed using Statistical Package for Social Sciences (SPSS) version 23.0 for Windows. The frequencies and mean values of the participants' sociodemographic variables were calculated. Sociodemographic and clinical variables of IBS and control groups were compared using independent t-tests and chi-square analysis. To test the relationship between anger, chronic pain, and depression, a Pearson's correlation matrix was calculated. A mediational model was used to suggest an indirect relationship between chronic pain and depression through linkages with anger. To test these models, a bootstrapping method developed by Preacher and Hayes for small sample sizes was used [16].

\section{RESULTS}

Seventy patients with IBS and 55 controls were enrolled. The means and standard deviations of all indicator variables for both groups are listed in Table I. The groups did not differ in gender, age, or level of education. The IBS group had 37 unemployed participants, whereas the control group had 3 , the difference being significant $(\mathrm{p}<0.001)$. Regarding the marital status, 22 IBS patients and 7 controls had no stable partner, the difference being significant $(\mathrm{p}=0.014)$. The $\mathrm{t}$-test for independent samples indicated that IBS patients had more severe STAXI-2 TANG $(\mathrm{p}=0.014)$ and AINDEX $(\mathrm{p}=0.046)$ compared to healthy individuals.

The mean of 36-SF-BP to measure pain in IBS group was $50.46 \pm 25.55)$. The BDI scale measured the severity of the depressive symptoms: the mean score was $14.30 \pm 9.45$. Based on BDI score, $58.6 \%$ IBS patients reported various degrees of depression: $35.7 \%$ had mild, $12.9 \%$ moderate, and $10 \%$ severe depression).

Chronic pain had a negative moderate correlation with depression $(r=-0.407 \mathrm{p}<0.001)$ (Fig. 1), and negative weak correlations with STAXI-2 TANG $(\mathrm{r}=-0.376, \mathrm{p}=0.023)$ and 
Table I. Sociodemographic and clinical data

\begin{tabular}{|c|c|c|c|}
\hline $\begin{array}{l}\text { Sociodemographic and } \\
\text { clinical variables }\end{array}$ & $\begin{array}{l}\text { IBS group } \\
(\mathrm{n}=70)\end{array}$ & $\begin{array}{c}\text { Control group } \\
\quad(\mathrm{n}=55)\end{array}$ & $\mathrm{p}$ \\
\hline \multicolumn{4}{|l|}{ Gender n, (\%) } \\
\hline Female & $21(30 \%)$ & $23(41.8 \%)$ & \multirow[t]{2}{*}{$0.170^{*}$} \\
\hline Male & $49(70 \%)$ & $32(58.2 \%)$ & \\
\hline Age (years $\pm \mathrm{SD}$ ) & $48.02 \pm 13.22$ & $46.12 \pm 10.27$ & $0.367^{\wedge}$ \\
\hline \multicolumn{4}{|l|}{ Marital status, n (\%) } \\
\hline Stable partner & $48(68.6 \%)$ & $48(87.3 \%)$ & \multirow[t]{2}{*}{$0.014^{*}$} \\
\hline No partner & $22(38.4 \%)$ & $7(12.7 \%)$ & \\
\hline \multicolumn{4}{|l|}{ Working status, $\mathrm{n}(\%)$} \\
\hline Employed & $33(47.1 \%)$ & $52(94.5 \%)$ & \multirow[t]{2}{*}{$<0.001^{\star}$} \\
\hline Unemployed & $37(52.9 \%)$ & $3(5.5 \%)$ & \\
\hline Education (years $\pm S D$ ) & $12.64 \pm 2.15$ & $13.16 \pm 2.67$ & $0.349^{\wedge}$ \\
\hline $\begin{array}{l}\text { STAXI-2-SANG } \\
(\text { mean } \pm \text { SD })\end{array}$ & $18.18 \pm 7.05$ & $16.92 \pm 6.23$ & $0.297^{\wedge}$ \\
\hline $\begin{array}{l}\text { STAXI-2-TANG } \\
(\text { mean } \pm \text { SD })\end{array}$ & $19.82 \pm 7.37$ & $16.12 \pm 5.49$ & $0.014^{\wedge}$ \\
\hline $\operatorname{AINDEX}($ mean $\pm \mathrm{SD})$ & $39.91 \pm 14.82$ & $35.70 \pm 12.12$ & $0.046^{\wedge}$ \\
\hline
\end{tabular}

STAXI-2-SANG: State-Anger (intensity of anger feelings at some point in time); STAXI-2-TANG Trait-Anger (disposition to feel anger); AINDEX Anger Expression Index; ${ }^{*}$ : Chi-square test; $\wedge$ : $\mathrm{t}$-test

AINDEX $(\mathrm{r}=-0.338, \mathrm{p}=0.048)$. There was no association between chronic pain and STAXI-2 SANG.

There were positive moderate correlations between depression and STAXI-2 SANG $(r=0.480, \mathrm{p}<0.001)$, depression and STAXI-2 TANG ( $\mathrm{r}=0.489, \mathrm{p}<0.001)$ (Fig. 2) and depression and $\operatorname{AINDEX}(\mathrm{r}=0.368, \mathrm{p}=0.002)$ (Fig. 3).

It was hypothesized that anger trait and anger expression would act as mediating variables between chronic pain and depression. The results from the mediational models showed that the regression coefficient for the total indirect effect for anger trait $\mathrm{B}=0.0027$ was significantly different from 0 (95\% CI $-0.0061,-0.0006)$ and for anger expression $\mathrm{B}=-0.0035$ was also significantly different from 0 (95\%, CI -.0095, -.0004) and therefore did support the effects of mediation. In other words anger trait and anger expression did mediate the relationship between chronic pain and depression.

\section{DISCUSSION}

This study aimed to investigate anger state, anger trait and anger expression style in a relatively small sample of IBS patients in order to address the gaps in the literature relating to the potential mediating effect of anger on the chronic paindepression relationship.

Our group of IBS patients showed higher scores of STAXI-2 TANG and lack of control of anger expression compared to healthy controls. Moreover, anger trait and anger expression mediated the relationship between pain and depression. The current findings also contribute to the research examining psychosocial processes underlying depression in IBS patients.

Our findings are consistent with previous research indicating a positive association between anger,clinical risk factors for IBS and chronic pain. Previous studies specified the role of anger in IBS: anger decreased antral motor activity in IBS patients and not in healthy controls; also anger suppression

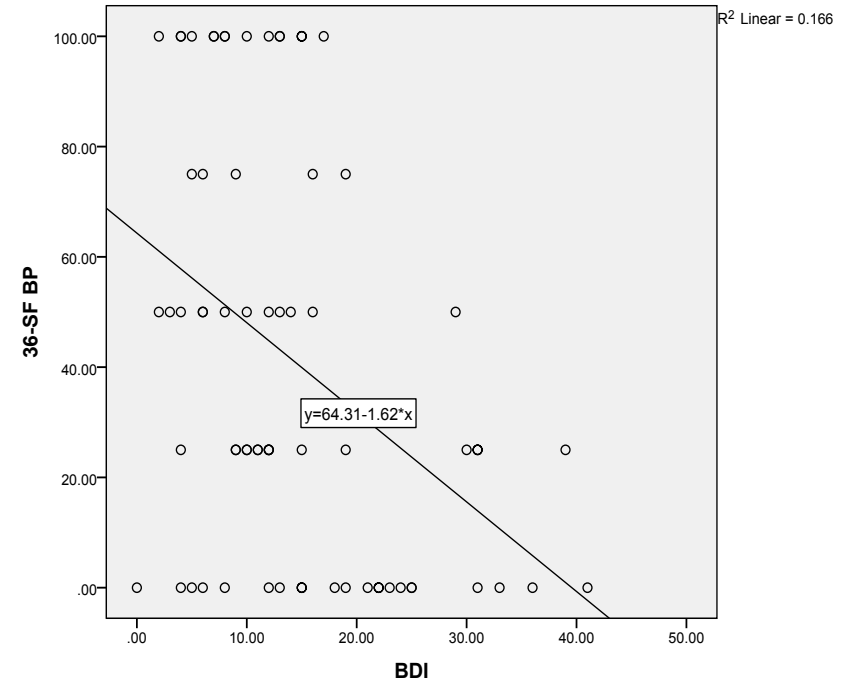

Fig. 1. Correlation between depression (BDI) and chronic pain (36SP BP).

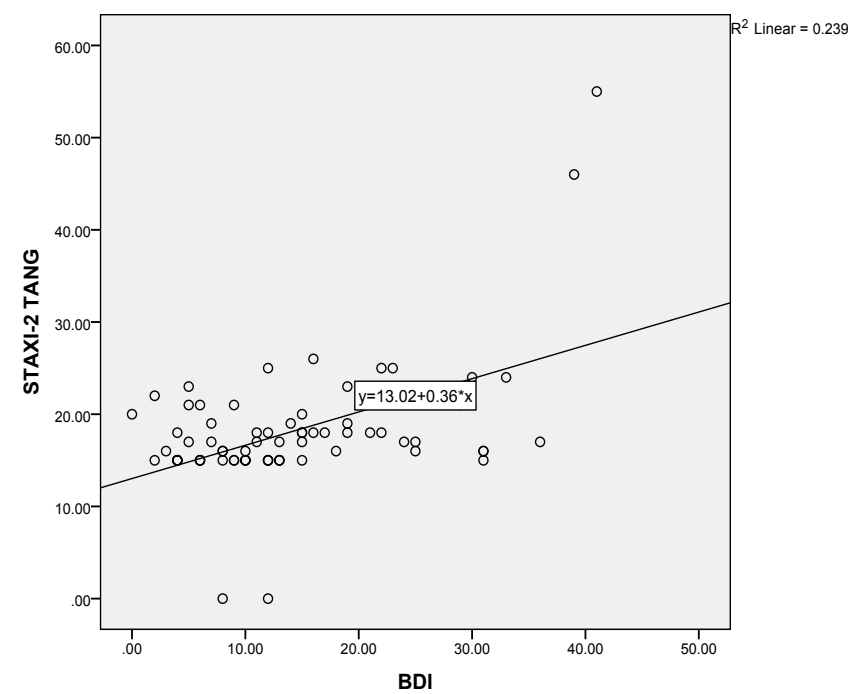

Fig. 2. Correlation between depression (BDI) and anger trait (STAXI-2 TANG).

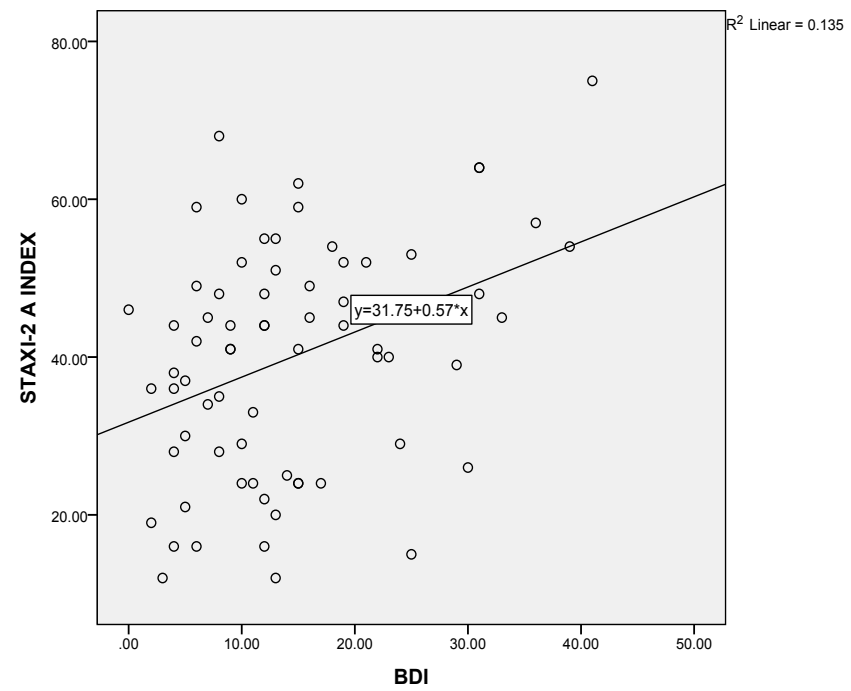

Fig. 3. Correlation between depression (BDI) and anger expression (STAXI-2 A INDEX). 
was associated with prolonged gastric emptying and delayed gut transit in the IBS population $[17,18]$. Another study suggested that IBS subtypes showed different symptomatic profiles in negative emotions, IBS patients with constipation being more psychologically distressed than IBS subjects with diarrhea [19]. Mechanisms involved in this association are complicated, and the present study was not designed to elucidate them.

Patients endorsing higher levels of pain reported significantly higher levels of STAXI-2-TANG and depression. Previous research reported that chronic pain was associated with depression, trait anger, and anger regulation style [20-22]. Depression is often associated with poor treatment adherence and a more intensive and expensive medical follow-up [23-25]. The association between depression and chronic pain was validated in several epidemiological studies [26, 27].

Our research has several limitations: eligible subjects were recruited from IBS patients referred to tertiary gastroenterology centers. They are likely to be individuals with more severe forms of IBS, who had been seeking treatment for many years, often with limited success. . Also, the small sample size could lead to an increase in the variability and could decrease the accuracy to detect differences between the two groups.

The results of this study should not be generalized to all IBS patients. However, we consider our study group representative for the IBS patients willing to engage with complex biopsychosocial interventions targeting negative emotions such as anger, anxiety, and depression (psychoeducation, psychotherapy, pharmacotherapy). This study was a cross-sectional examination of participants with IBS and healthy controls. For that reason, causal inferences in terms of the directionality of examined effects cannot be made. Given the limitations of the cross-sectional analysis, future longitudinal studies, where the stability of constructs can be better measured are warranted.

\section{CONCLUSIONS}

Our results suggest that anger trait and anger expression style may explain the impact of perceived pain on depressive symptoms in IBS patients. Greater emphasis should target the psychosocial factors in IBS patients. Complex psychological interventions targeting negative emotions in order to improve gastrointestinal symptoms in IBS patients, should be a standard treatment.

Conflicts of interest: The authors have no conflict of interest to report.

Authors' contributions: M.F.S. and T.S.B. the concept and design of the study; M.F.S. and C.P. data acquisition; O.C. statistical analysis. All authors analyzed the data, critically revised the manuscript, approved the final version.

\section{REFERENCES}

1. Chey WD, Kurlander J, Eswaran S. Irritable bowel syndrome: a clinical review. JAMA 2015;313:949-958. doi:10.1001/jama.2015.0954

2. Frändemark $\AA$, Törnblom H, Jakobsson S, Simrén M. Work Productivity and Activity Impairment in Irritable Bowel Syndrome (IBS): A
Multifaceted Problem. Am J Gastroenterol 2018;113:1540-1549. doi:10.1038/s41395-018-0262-x

3. Doshi JA, Cai Q, Buono JL, et al. Economic burden of irritable bowel syndrome with constipation: a retrospective analysis of health care costs in a commercially insured population. J Manag Care Spec Pharm 2014;20:382-390. doi:10.18553/jmcp.2014.20.4.382

4. Tanaka Y, Kanazawa M, Fukudo S, Drossman DA. Biopsychosocial model of irritable bowel syndrome. J Neurogastroenterol Motil 2011;17:131-139. doi:10.5056/jnm.2011.17.2.131

5. Moloney RD, O’Mahony SM, Dinan TG, Cryan JF. Stress-induced visceral pain: toward animal models of irritable-bowel syndrome and associated comorbidities. Front Psychiatry 2015;6:15. doi:10.3389/fpsyt.2015.00015

6. Camilleri M. Physiological underpinnings of irritable bowel syndrome: neurohormonal mechanisms. J Physiol 2014;592:2967-2980. doi:10.1113/jphysiol.2014.270892

7. Keefer L, Palsson OS, Pandolfino JE. Best Practice Update: Incorporating Psychogastroenterology Into Management of Digestive Disorders. Gastroenterology 2018;154:1249-1257. doi:10.1053/j.gastro.2018.01.045

8. Greenwood KA, Thurston R, Rumble M, Waters SJ, Keefe FJ. Anger and persistent pain: current status and future directions. Pain 2003;103:1-5. doi:10.1016/S0304-3959(03)00132-5

9. Burns JW, Bruehl S, Chont M. Anger regulation style, anger arousal and acute pain sensitivity: evidence for an endogenous opioid "triggering" model. J Behav Med 2014;37:642-653. doi:10.1007/s10865-013-9511-Z

10. Adams LM, Turk DC. Psychosocial factors and central sensitivity syndromes. Curr Rheumatol Rev 2015;11:96-108. doi:10.2174/15733 97111666150619095330

11. Fadgyas-Stanculete M, Dumitrascu DL, Pojoga C, Nedelcu L. Coping strategies and dysfunctional cognitions as predictors of irritable bowel syndrome diagnosis. Journal of Evidence-Based Psychotherapies 2015;15:113-122.

12. Zhang QE, Wang F, Qin G, et al. Depressive symptoms in patients with irritable bowel syndrome: a meta-analysis of comparative studies. Int J Biol Sci 2018;14:1504-1512. doi:10.7150/ijbs.25001

13. Muscatello MR, Bruno A, Scimeca G, Pandolfo G, Zoccali RA. Role of negative affects in pathophysiology and clinical expression of irritable bowel syndrome. World J Gastroenterol 2014;20:7570-7586. doi:10.3748/wjg.v20.i24.7570

14. Surdea-Blaga T, Baban A, Nedelcu L, Dumitrascu DL. Psychological Interventions for Irritable Bowel Syndrome. J Gastrointestin Liver Dis 2016;25:359-366. doi:10.15403/jgld.2014.1121.253.ibs

15. Gerson CD, Gerson MJ, Chang L, et al. A cross-cultural investigation of attachment style, catastrophizing, negative pain beliefs, and symptom severity in irritable bowel syndrome. Neurogastroenterol Motil 2015;27:490-500. doi:10.1111/nmo.12518

16. Preacher KJ, Hayes AF. SPSS and SAS procedures for estimating indirect effects in simple mediation models. Behav Res Methods Instrum Comput 2004;36:717-731. doi:10.3758/bf03206553

17. Welgan P, Meshkinpour H, Ma L. Role of anger in antral motor activity in irritable bowel syndrome. Dig Dis Sci 2000;45:248-251. doi:10.1023/a:1005487821063

18. Bennett EJ, Evans P, Scott AM, et al. Psychological and sex features of delayed gut transit in functional gastrointestinal disorders. Gut 2000;46:83-87. doi:10.1136/gut.46.1.83

19. Muscatello MR, Bruno A, Pandolfo G, et al. Depression, anxiety and anger in subtypes of irritable bowel syndrome patients. J Clin Psychol Med Settings 2010;17:64-70. doi:10.1007/s10880-009-9182-7

20. Hampton AJ, Hadjistavropoulos T, Gagnon MM, Williams J, Clark D. The effects of emotion regulation strategies on the pain experience: 
a structured laboratory investigation. Pain 2015;156:868-879. doi:10.1097/j.pain.0000000000000126

21. Scott W, Sullivan M. Perceived injustice moderates the relationship between pain and depressive symptoms among individuals with persistent musculoskeletal pain. Pain Res Manag 2012;17:335-340. doi:10.1155/2012/501260

22. Slavin-Spenny O, Lumley MA, Thakur ER, Nevedal DC, Hijazi AM. Effects of anger awareness and expression training versus relaxation training on headaches: A randomized trial. Ann Behav Med 2013;46:181-192. doi:10.1007/s12160-013-9500-z

23. Grenard JL, Munjas BA, Adams JL, et al. Depression and medication adherence in the treatment of chronic diseases in the United States: a meta-analysis. J Gen Intern Med 2011;26:1175-1182. doi:10.1007/ s11606-011-1704-y
24. Dempe C, Jünger J, Hoppe S, et al. Association of anxious and depressive symptoms with medication nonadherence in patients with stable coronary artery disease. J Psychosom Res 2013;74:122-127. doi:10.1016/j.jpsychores.2012.12.003

25. Sin NL, DiMatteo MR. Depression treatment enhances adherence to antiretroviral therapy: a meta-analysis. Ann Behav Med 2013;47:259269. doi:10.1007/s12160-013-9559-6

26. Dueñas M, Ojeda B, Salazar A, Mico JA, Failde I. A review of chronic pain impact on patients, their social environment and the health care system. J Pain Res 2016;9:457-467. doi:10.2147/JPR.S105892

27. Lee HJ, Choi EJ, Nahm FS, Yoon IY, Lee PB. Prevalence of unrecognized depression in patients with chronic pain without a history of psychiatric diseases. Korean J Pain 2018;31:116-124. doi:10.3344/kjp.2018.31.2.116 